\title{
IoT Technologies Systems on Medical Monitoring and Management Systems with a Real-Time ECG Signal Transmission Monitoring Algorithm
}

\author{
Aaron Don M. Africa, Bryon Gil Cajayon, Adrian Alano \\ De La Salle University, Manila \\ 2401 Taft Ave., Malate, Manila 1004, \\ Philippines, aaron.africa@dlsu.edu.ph
}

\begin{abstract}
The concept of the Internet of Things (IoT), revolves around connecting a data network that extends beyond the standard computers and smart devices, into simpler everyday objects, such as lighting, air conditioning, medical equipment, and street signs, which allow them to be remotely monitored and controlled. Even though this concept of having a network of intelligent devices has been discussed for as early as the 1980s, it hasn't seen much traction and implementation until recent years, thus, still making it an unfamiliar concept to many. With having a bunch of remotely controllable and accessible different everyday objects, this concept can be used in emergency situations, in order to reduce the impact of such occurrences, and hasten recovery. This study focuses to explore the different possible approaches in using IoT for hospital and healthcare management and specific examples of possible real-life scenario applications. By doing so, the paper was able to present possible parameters of measurement between different IoT healthcare networks to compare and determine the best possible implementation to use.
\end{abstract}

Key words: Data Networks, Computer Networks, Internet of Things, Smart Devices, Healthcare Management.

\section{INTRODUCTION}

Hospitals and healthcare use various devices to check their patient's wellbeing to infer or diagnose them. Due to this hospitals are divided in various departments to house this devices creating distance from the Doctors that would need the output from the devices or equipment and the actual equipment for most healthcare institutions this is supplemented by a network within the hospital that is still manually recorded on a single network [1]. However, Hospitals sometimes overlook significant data in terms of diagnosis causing a delay in creating or making a diagnosis in some scenarios this may be disadvantageous if it is to say an emergency. Solutions that aid doctors in terms of diagnosis are already being used however, the entire process of gathering information from the patient is still followed and done manually [2].

Internet of Things (IoT), is simply a term used to describe a system of interconnected devices, big or small, used for big computations, or used simply for tracking or as a sensor, which is differentiated by providing each one with a unique identifier (UID), and can transfer data by itself within the network $[3,4,5,6]$.

In terms of computer networks, the Internet of Things involves a huge data network involving multiple smart devices interconnected with one another [7,8]. IoT can be implemented similar to a computer network, wherein each host can be given an IP address as an identifier, and data can be exchanged from one device to another [9].

Technology has advanced so far, that a smartphone can do so much more than the traditional call and text $[10,11]$. Thus, by applying a similar approach to smaller everyday objects found lying around, we can integrate machines into our lives further.

IoT is an arising field, yet we don't see much use and application [12]. Usage in emergency management is important, as it allows us to gather data and control various devices together for quick impact removal and/or reduction remotely via control systems $[13,14]$. In these situations, one of the common problems is the transfer of accurate information between the individuals, and this can be addressed through the IoT system. These devices can be able to create a unified system which will be able to report multiple metrics about an emergency efficiently and send this information to a response service. Though there have been studies about the applications of this system, there has not been an organized approach developed to determine which of these is the best $[15,16]$. In this paper, the researchers will try to address this problem by comparing various applications of IoT in terms of different factors. This paper will also be able to determine which studies are most efficient and realistic for individuals. 
Aaron Don M. Africa et al., International Journal of Emerging Trends in Engineering Research, 8(5), May 2020, 1897 - 1901

\section{METHODOLOGY}

By doing a study on papers, we can come across IoT Concepts and terms, which are probably different, or normally not seen in normal computer networks. At the same time, by surveying IEEE papers, we get to explore and read on about current advancements in the said field.

With this in mind, the survey was done by looking into several papers that provide concepts on integrating the concept of the Internet of Things to handling and/or monitoring hospital or healthcare situations. In this study, the papers that were used as a basis were mostly taken from published IEEE Transactions or Journals. Papers were then tabulated to compare certain parameters from different studies. In this survey, the researchers chose to focus on distance, energy consumption, latency, the privacy of connections, and possible nodes that the network can support, becausein IoT networks, especially one for emergencies, these common factors prove to be essential in determining the network's limits in responding to different scenarios. The distance covered by the network is important in determining the range for the system. Energy consumption is also important to monitor how much energy used. The latency should also be thought of since time and delay are important in emergencies. The type of connection will help the privacy of connections, and possible nodes.

\section{RESULTS AND DISCUSSION}

\subsection{Summary of Papers}

The summary of the survey is tabulated as in Table 1.

Table 1: Survey Tabulation

Paper Reference: (F.Wu, et al, 2019) [17]

Title: Design and Implementation of a Wearable Sensor Network System for IoT-Connected Safety and Health Applications.

Connection (Private or public): Connections implemented were hosted privately by Digita 1Ocean a cloud service provider.

Possible nodes or people connected: Nodes in this implementation are mainly people. One of the elements for an IoT system is the environment where all primitives of IoT in a specific network operate in and nodes are included.

Summary: The paper discusses the implementation of WBAN (Wearable Body Area Network) to create an IoT network system that is able to monitor both physiological and environmental data forming a network of wearable sensors attached to workers' body and provide invaluable information to the system operator and workers for safety and health monitoring.
Paper Reference: (Farhat, et al, 2018) [18]

Title: Medical Equipment Efficient Failure Management in IoT Environment.

Connection: Connections implemented was hosted locally on their own servers and devices.

Possible nodes or people connected: Nodes in this implementation involved medical equipment. Connecting all the equipment for better synchronization of usage.

Summary:The article monitored various medical equipment with the use of different services to provide and compare historical data which is then subjected to logistic regression to be able to predict the point of failure and maintenance of medical types of equipment connected to the network.

Paper Reference: (Ranjbar, et al , 2019) [19]

Title: Design of an IoT-Based System for Smart Maintenance of Medical Equipment.

Connection: Connections are hosted privately with public access using a local server

Possible nodes or people connected: The implementation place nodes on the equipment besides they granted access to the equipment company supplier to their servers by putting necessary controls for the admins of the servers.

Summary: The research focuses on different aspects of moving essential information for doctors as well as their medtech staff for maintenance and ease of communication. The device connects various medical equipment for monitoring of its use or patience as well as the integrity of the equipment.

Paper Reference:(Djelouat, et al, 2020) [20]

Title: Real-time ECG monitoring using compressive sensing on a heterogeneous multicore edge-device.

Connection: The paper would like to implement their system into a multiplatform structure thus restrictions for connection depends on the platforms that are going to connect.

Possible nodes or people connected:The possible nodes discussed in the paper are through edge devices and focused on wearable devices that exhibit multiplatform hierarchy also the paper presented a tree structure with wearable devices as leaves.

Summary:The paper proposed a solution in terms of creating a wearable healthcare monitoring system having real-time vital signs as well as containing healthcare records as well as other pseudo vital signs in addition the paper proposed that their device be implemented in a multiplatform tree architecture having the devices as leaves. 
Paper Reference:(Arefin, et al, 2019) [21]

Title: The Basics of Healthcare IoT: Data Acquisition, Medical Devices, Instrumentations and Measurements.

Connection: The article provided various sensors or devices and strategies in implementing an IoT for the use of Healthcare that can either be private or public

Possible nodes or people connected:The article discussed implementation on edge devices such as wearable devices, medical equipment, and specialized sensors meaning that the nodes are proportional to the structure to be implemented by consumers or users.

Summary: The article provided various sensors and strategies that can be implemented in IoT that are specifically focused on the field of Healthcare sensors that are both capable of being used in a smaller form factor as well as able to provide accurate information are highlighted in this article as well.

\subsection{Discussion}

To compare the different studies viewed by the group, we looked at certain parameters as shown in the table above. These parameters are chosen to assess the scalability and overall integrity of the implementations by the researchers.

Now, for connection privacy, most of the papers reviewed used a local private server, however, the paper by Ranjbar proposed a system that is privately and locally hosted that can grant controlled access to public entities. Also, using cloud services by DigitalOcean can ensure that the server will be properly maintained and most of the time available for distribution of information to its nodes or edge devices.

This system was proposed to be built around a huge IoT network, wherein users would send sensitive information from their wearable devices to use them to better monitor different situations, thus making sure of the security of the network.

Lastly, for possible network nodes, most of the paper proposed a point to point connection from the server to the edge devices, however, Djelout proposed a multiplatform structure to better manage the traffic a server would receive as well as to be able to provide process data that is more valuable for record-keeping than data that is raw.

Overall, it seems that a very useful study by Arefin because it provided up to date information in terms of the possible devices and applications of IoT in the field of healthcare while giving liberty in the selection of the parameters that are set by this review. The Healthcare IoT System is shown in Figure 1.

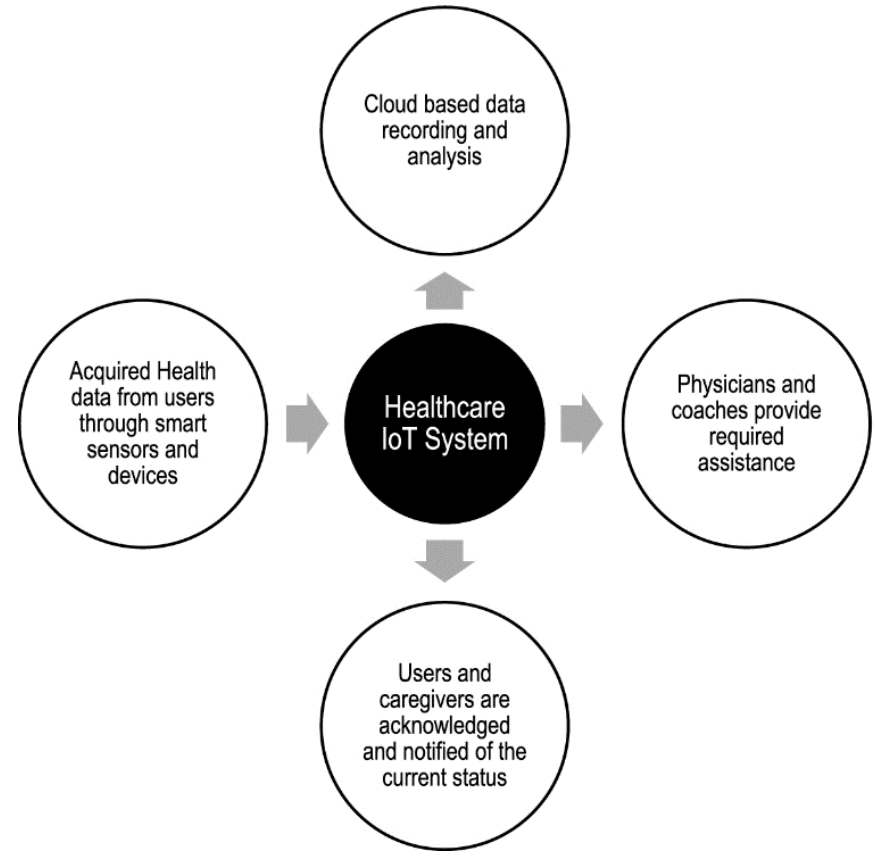

Figure 1: Healthcare IoT Systems [21]

After looking at various studies, it seems that studies need to detail more on the distance limits of the devices in the network. If the proposed IoT system is expandable, the study can be further improved by detailing the layout of the network and how far a certain set of devices range are, and how each device adds to the range of the network for most of the devices proposed are wearable devices it is prone to go out of bounds due to the movement of the users if they are not to be confined in a specific space like discussed by the paper of $\mathrm{Wu}$ however if the ideal setup of the devices is that of Djelout distance limits must be detailed out.

Also, regarding the studies that the researchers viewed, it did not specify a limit to the number of nodes to the network, and the possible workarounds around it. This could also be taking into account the limits of the devices involved in maintaining the network, such as in a maximum number of connections per access point, aside from the distance factor, since after all, an IoT network could involve a huge number of different devices that are much unlike traditional networks. And also, other studies can choose to experiment on actual power consumption or give a quantitative theoretical value, since this is important in determining how long the healthcare system would last in situations where line power is unavailable.

On the other hand, latency is also important, so systems should also study and give a quantitative amount on the amount of delay between transmission processing and reception of data in the system, and whether it is hard real-time, or soft real-time system, to act as a benchmark to understand limitations of proposed systems better, and allow further studies to research for possible improvements on this matter with further advancements in technology. 


\section{REAL-TIME ECG SIGNAL TRANSMISSION MONITORING ALGORITHM}

In regards to the Internet of Things (IoT) and because it is a concept that involves connecting multiple smart devices a Real Time ECG Signal can use this concept. It can turn quantized signals into encoded signals that can be transmitted.
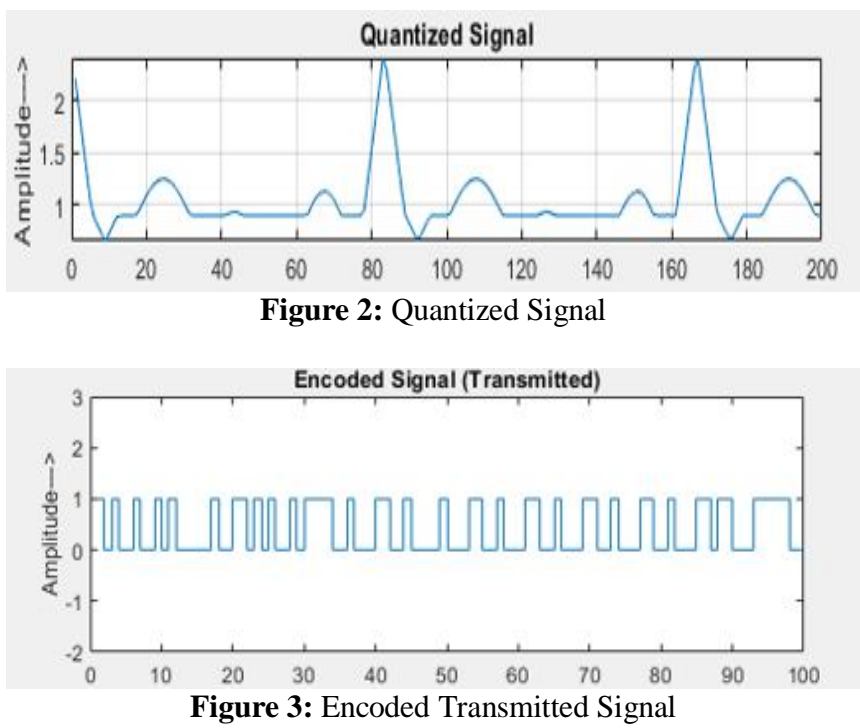

In light of the utilization of a FSK adjusting plan, it was discovered that the proposed framework accomplishes its objective of ongoing transmission and gathering of ECG signals. Although the MATLAB recreation requires some preparing time, the framework might be executed utilizing simple circuits. The obtaining of the ECG signal is frequently done utilizing restrictive gear, which presents no deferral. The low-pass channel following the securing segment presents a unimportant postpone that relates to an under 180-degree stage move whenever actualized utilizing RC segments.

The enhancer might be executed utilizing operational intensifiers, which present insignificant deferral. The beat code modulator yields equally divided examples in time. The FSK beneficiary and demodulator, when executed analogically, present no postponement. The transmission of radio signals through any medium sees some postponement because of the separation between the transmitter and recipient; be that as it may, this can't be maintained a strategic distance from in many frameworks.

\section{CONCLUSION}

Internet of Things (IoT) is a concept that involves connecting multiple smart devices other than computers or smartphones to a huge network. These devices can include small wearables, sensors placed around a facility. A lot of healthcare institutions can benefit from the implementation of the devices and papers in this review without touching the code of ethics of Doctors and will make them work efficiently such as IoT implementations of an entire hospital Doctors will gain access to the medical equipment that are being used to monitor their patients and can have real time and recorded comparisons on their patients providing micromanagement of resources and diagnosis. In regard to data optimization the Rough Set Theory can be used [22,23]. Making it into a computer system, it can follow the design of [24].

With the amount of available studies regarding IoT devices for healthcare devices, many would present and experiment with new concepts to be implemented, but then never dove down on some of the specific limitations of the study itself. In this survey, the researchers were able to point out some characteristics that are essential to choosing an IoT network to be implemented for healthcare handling and management, which can also act as benchmarks to improve implementations upon one another. At the same time, this study was also able to point out common characteristics about current studies on emergency IoT networks, that can be further improved, and better measured in the future.

\section{REFERENCES}

[1] A. Rusydi, S. Palutturi, N. Noor and S. Pasinringi, "The comparison of good corporate governance on human resources performance in regional general hospital of Labuang Baji and Ibnu Sina hospital Makassar, South Sulawesi," Enfermería Clínica.Vol. 30, No. 2, pp. 482-486, 2020. https://doi.org/10.1016/j.enfcli.2019.07.143

[2] O. Yang, M. Chan, T. Cheng and J. Yong, "Cream skimming: Theory and evidence from hospital transfers and capacity utilization," Journal of Economic Behavior \& Organization.Vol. 173, pp. 68-87, 2020. https://doi.org/10.1016/j.jebo.2020.03.024

[3] D. Jiang, "The construction of smart city information system based on the Internet of Things and cloud computing." Computer Communications. Vol. 150, pp.158-166, 2020. https://doi.org/10.1016/j.comcom.2019.10.035

[4] D. Goad, A. Collins and U. Gal, "Privacy and the Internet of Things-An experiment in discrete choice." Information \& Management. 2020. https://doi.org/10.1016/j.im.2020.103292

[5] Y. Xia, "Resource scheduling for piano teaching system of internet of things based on mobile edge computing," Computer Communications. Vol. 158, pp. 73-84, 2020.

[6] C. Uy and A. Africa, "Development of a cost-efficient waste bin management system with mobile monitoring and tracking." International Journal of Advanced Trends in Computer Science and Engineering. Vol. 8, No. 2, pp 319-327, 2019. https://doi.org/10.30534/ijatcse/2019/35822019

[7] B. Diène, J. Rodrigues, O. Diallo, E.Ndoye, and V. Korotaev, "Data management techniques for Internet of Things." Mechanical Systems and Signal Processing. Vol. 138, 2020. 
Aaron Don M. Africa et al., International Journal of Emerging Trends in Engineering Research, 8(5), May 2020, 1897 - 1901

[8] A. Rhayem, M. AhmedMhiri, and F. Gargouri, "Semantic Web Technologies for the Internet of Things: Systematic Literature Review.” Internet of Things. 2020. https://doi.org/10.1016/j.iot.2020.100206

[9] H. Zhang and X. Lu, "Vehicle communication network in intelligent transportation system based on internet of things," Computer Communications.2020.

[10] A. Africa, F. Espiritu, C. Lontoc, and R. Mendez, "The integration of computer systems into the expansive field of video games." International Journal of Advanced Trends in Computer Science and Engineering. Vol. 8, No. 4, pp. 1139-1145, 2019. https://doi.org/10.30534/ijatcse/2019/22842019

[11]A. Africa, G. Ching, K. Go, R. Evidente, J. Uy, "A comprehensive study on application development software systems." International Journal of Emerging Trends in Engineering Research. Vol. 7, No. 8, pp 99-103, 2019. https://doi.org/10.30534/ijeter/2019/03782019

[12]X. Liu, "Research on intelligent visual image feature region acquisition algorithm in Internet of Things framework," Computer Communications. Vol. 151, pp. 299-305, 2020. https://doi.org/10.1016/j.comcom.2020.01.008

[13] A. Africa, P. Arevalo, A. Publico, and M. Tan, "Linear system interconnections, steady-state analysis and stability theory." International Journal of Advanced Trends in Computer Science and Engineering. Vol. 8, No. 4, pp. 1395-1398, 2019. https://doi.org/10.30534/ijatcse/2019/56842019

[14]A. Africa, P. Arevalo, A. Publico, and M. Tan. "A comprehensive study of the functions and operations of control systems." International Journal of Advanced Trends in Computer Science and Engineering. Vol. 8, No. 3, pp. 922-926, 2019. https://doi.org/10.30534/ijatcse/2019/89832019

[15] Y. Ma and G. Guo, "Intelligent algorithm of geotechnical test data based on Internet of Things," Computer Communications. Vol. 158, pp. 32-38, 2020.

https://doi.org/10.1016/j.comcom.2020.04.028

[16]L. Torrizo and A. Africa, "Next-hour electrical load forecasting using an artificial neural network: Applicability in the Philippines," International Journal of Advanced Trends in Computer Science and Engineering. Vol. 8, No. 3, pp. 831-835, 2019. https://doi.org/10.30534/ijatcse/2019/77832019

[17]F. Wu, T. Wu, and M. R. Yuce, "Design and Implementation of a Wearable Sensor Network System for IoT-Connected Safety and Health Applications," 2019 IEEE 5th World Forum on Internet of Things (WF-IoT). 2019. https://doi.org/10.1109/WF-IoT.2019.8767280

[18] J. Farhat, A. Shamayleh, and H. Al-Nashash, "Medical equipment efficient failure management in IoT environment," 2018 Advances in Science and Engineering Technology International Conferences (ASET). 2018. https://doi.org/10.1109/ICASET.2018.8376911

[19]E. Ranjbar, R. G. Sedehi, M. Rashidi, and A. A. Suratgar, "Design of an IoT-Based System for Smart Maintenance of Medical Equipment," 2019 3rd International Conference on Internet of Things and Applications (IoT). 2019.

[20]H. Djelouat, M. A. Disi, I. Boukhenoufa, A. Amira, F. Bensaali, C. Kotronis, E. Politi, M. Nikolaidou, and G. Dimitrakopoulos, "Real-time ECG monitoring using compressive sensing on a heterogeneous multicore edge-device," Microprocessors and Microsystems. Vol. 72, p. 102839, 2020. https://doi.org/10.1016/j.micpro.2019.06.009

[21]A. Arefin, K. Nahiyan, and M. Rabbani, "The Basics of Healthcare IoT: Data Acquisition, Medical Devices, Instrumentations and Measurements," A Handbook of Internet of Things in Biomedical and Cyber Physical System Intelligent Systems Reference Library. pp. 1-37, 2019. https://doi.org/10.1007/978-3-030-23983-1_1

[22] A. Africa, "A rough set-based data model for heart disease diagnostics." ARPN Journal of Engineering and Applied Sciences. Vol. 11, No. 15, pp. 9350-9357, 2016.

[23] M. Abdolrazzagh-Nezhad, H. Radgohar and S. Salimian, "Enhanced cultural algorithm to solve multi-objective attribute reduction based on rough set theory." Mathematics and Computers in Simulation. Vol. 170,pp.332-350, 2020. https://doi.org/10.1016/j.matcom.2019.11.005

[24]A. Africa, T. Dolores, M. Lim, L. Miguel, and V. Sayoc. "Understanding logical reasoning through computer systems." International Journal of Emerging Trends in Engineering Research. Vol. 8, No. 4, pp 1187-1191, 2020. 